\title{
Réseaux d'eau potable : coûts actualisés. Application à l'A.E.P. Buenos Aires
}

\author{
Drinking water system cost Discounting. \\ The example of Buenos Aires Drinking Water Supply
}

par Etienne Frossard

Aguas Argentinas, Lyonnaise des Eaux

\author{
Christian Criado
}

Safege Argentine, Safege-Montgomery Watson

Christian Coite

BRL Ingénierie

It takes pumping stations, supply and distribution mains and regulation facilities to deliver drinking water from the treatment plant to the consumer. Often, the dimensions of these water works are defined by nonspecified rules related to velocity or pressure in the pipes. This type of rule can considerably raise the cost of DWS projects.

Current value conversion and optimization studies on the large conveyance lines in the Buenos Aires Drinking Water Supply System show that it is possible to make savings on investments and on energy. They also show that further technical improvement can increase the reliability of the conveyance facilities.

\section{PROBLÉMATIQUE}

Comme dans tous les projets hydrauliques, le maîtred'œuvre se doit d'étudier et de mettre en balance toutes les composantes économiques qui sont :

- le coût des investissements (conduites, réservoirs de stockage et stations de pompage),

- le coût d'énergie,

- le coût de maintenance, exploitation et renouvellement.

Il est bien connu que tous ces éléments varient à l'inverse les uns des autres. Plus l'investissement sera élevé, c'est-àdire plus on s'attachera à choisir les équipements les plus fiables (en général aussi les plus chers), plus seront réduits les frais de maintenance et de renouvellement ultérieur.

Plus les conduites seront de grand diamètre, plus les stations de pompage seront de puissance réduite et donc plus le coût d'énergie sera réduit.

Enfin, lorsqu'existe une tarification d'énergie pénalisant fortement les pompages en heures de pointe, il peut être intéressant de stocker l'eau pendant ces périodes.

Lors de l'établissement d'un projet, l'Ingénieur conseil peut donc se poser la question «la solution proposée est-elle celle qui garantit à mon client un projet optimisé ? ».
Cette question est essentielle en A.E.P. (Adduction d'eau potable), car les installations les plus courantes fonctionnent pratiquement en continu toute l'année et le produit est vendu à un prix (hors taxes) relativement bas.

Pourtant curieusement, cette notion d'optimisation reste encore peu utilisée, les ingénieurs se contentant souvent de règles empiriques issues de la bibliographie ou de l'usage propre au bureau d'étude.

On verra dans l'étude ci-après que des économies très sensibles peuvent être faites, grâce à une optimisation des caractéristiques des réseaux d'adduction et on verra l'application qui a pu en être faite pour les réseaux de l'A.E.P. de Buenos Aires.

\section{L'OPTIMISATION DES RÉSEAUX D'ADDUCTION}

Si l'on prend un cas d'école, celui d'une conduite reliant une station de pompage à un point de livraison, la ligne piézométrique prend les allures de la figure 1 , selon que l'on considère des diamètres de plus en plus grands. 


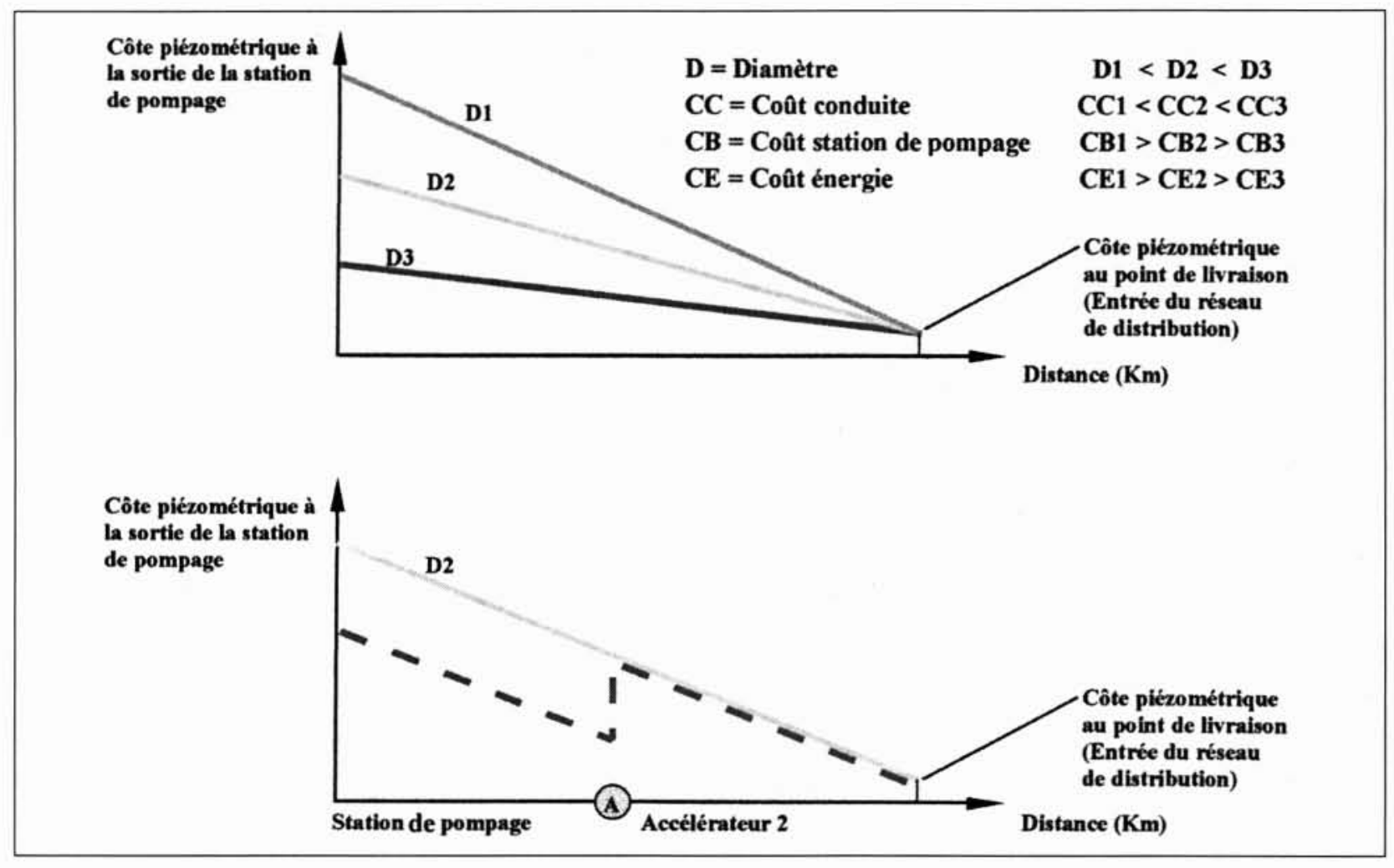

1. Lignes piézométriques en fonction du diamètre.

On conçoit que plus le diamètre sera grand, plus la HMT (hauteur moyenne totale) de la station sera réduite et avec elle la consommation d'énergie.

On notera, car nous l'utiliserons dans les chapitres ultérieurs, que la cote piézométrique nécessaire peut être fournie par une seule station en tête, ou répartie entre cette dernière et un accélérateur implanté en un point quelconque de la conduite.

Bien entendu, plus le diamètre de la conduite sera grand, plus les frais de maintenance et de renouvellement de cette dernière et des vannes associées sera grand. A contrario les frais de maintenance et de renouvellement de la station de pompage seront réduits.

L'élément le plus complexe à définir est le coût d'énergie. En général on se contente d'un coût au $\mathrm{kWh}$ moyen, alors que dans les pays évolués la complexité de la tarification devrait obliger à un calcul complet. Par exemple dans le tarif EDF ci-joint, on peut constater que le coût de l'énergie varie dans un rapport de 1 à 5 entre Heure Creuse d'Eté, et Heure de Pointe d'Hiver.

Dans un calcul complet, on considère les puissances souscrites et les volumes consommés dans chaque période tarifaire. Si l'on veut être encore plus rigoureux, on doit également considérer la progression des volumes d'une année à l'autre.

Afin de mettre en balance des coûts d'investissements (en grande partie faits l'année zéro) avec les coûts apparaissant durant toute la vie du projet (énergie, maintenance et renouvellement) on recourt à un calcul d'actualisation. Celui-ci consiste à regrouper toutes les dépenses sur la durée de vie d'un ouvrage la première année.

Le taux d'actualisation doit être un juste équilibre entre le taux des prêts bancaires et le taux d'inflation, mais il peut être pondéré pour tenir compte des orientations de politique économique en matière de planification et développement et la réalité des opportunités financières qui s'offrent à l'investisseur.

Si l'on dessine sur un graphe les coûts actualisés, résultants de tous ces éléments en fonction du diamètre de la conduite, on aboutit à la courbe en cloche inversée bien connue (fig. 2).

Cette courbe présente un minimum correspondant au diamètre optimal. En fait, en général deux ou trois diamètres peuvent être élus au titre de diamètre optimal, ce qui laisse une certaine latitude au maitre d'ouvrage, pour privilégier le premier investissement ou la consommation d'énergie.

Le problème est évidemment plus complexe dès lors que l'on s'adresse à un réseau multiple desservant de nombreux points de livraison, eux-mêmes ayant des pressions d'entrée différentes.

Dans ce cas, seule l'informatique peut aider l'ingénieur conseil. La méthode que nous avons utilisée est celle de Labye. Elle consiste, à partir d'une cote piézométrique en tête de réseau, à déterminer la meilleure combinaison de dia-

Tableau 1. - Tarif vert B Base.

\begin{tabular}{|c|c|c|c|c|c|c|c|c|c|}
\hline \multirow{3}{*}{$\begin{array}{c}\text { Barème } \\
\text { du } 20 / 04 / 1997\end{array}$} & \multirow{3}{*}{$\begin{array}{l}\text { Prime fixe } \\
\text { annuelle } \mathrm{f} / \mathrm{kW}\end{array}$} & \multicolumn{8}{|c|}{ Prix de l'énergie (c/kWh) } \\
\hline & & \multicolumn{5}{|c|}{ Hiver et demi saison } & \multicolumn{3}{|c|}{ Eté } \\
\hline & & PTE & $\mathrm{HPH}$ & HPD & $\mathrm{HCH}$ & HCD & HPE & HCE & JA \\
\hline LU & 337,68 & 62,24 & 47,26 & 31,18 & 30,22 & 20,10 & 20,35 & 12,15 & 10,33 \\
\hline
\end{tabular}


2. Evolution du coût total actualisé.

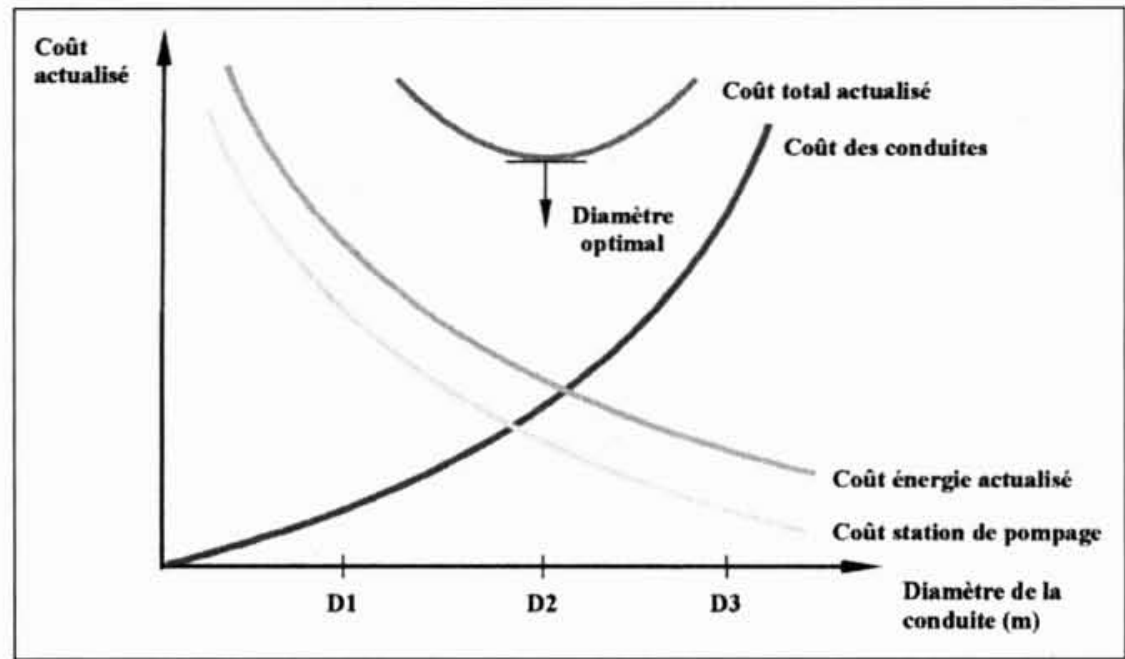

mètre des conduites, qui permettent de garantir la cote nécessaire en extrémité de chaque réseau. Avec un tableau de prix par diamètre, on obtient ainsi une courbe :

\section{Coût des conduites $=f$ (cote piézométrique)}

Pour chaque cote piézométrique, on peut calculer, comme précédemment, les coûts de la station de pompage et d'énergie.

Ici également on obtient une courbe en cloche inversée présentant un minimum qui est la cote piézométrique optimale en tête de réseau. A cette cote est associée une combinaison de diamètres sur les différentes branches (fig. 3).

Evidemment, à une telle méthode peuvent être associées toutes les contraintes souhaitées (vitesses mini et maxi, longueur minimale pour un certain diamètre, etc...).

\section{III —CHOIX DU MODE DE RÉGULATION DU SYSTÈME HYDRAULIQUE}

\subsection{Régulation à l'entrée du réseau de distribution}

Lorsqu'un projet comporte des adductions de grandes longueurs, il est usuel de prévoir, à l'amont du réseau de distribution, un réservoir surélevé.

Ce réservoir peut avoir plusieurs rôles (régulation de fonctionnement des pompes, équilibre, stockage de pointe); mais le plus important est la sécurité qu'il peut apporter en cas de panne sur les captages, stations de pompage ou réseaux amonts.

En contrepartie de ces avantages, on peut noter :

- le coût d'un tel ouvrage (investissement, entretien),

- le côté esthétique, la difficulté d'intégration dans un paysage urbain dense,

- le rôle de sécurité très relatif dans des grandes agglomérations $\left(1000 \mathrm{~m}^{3}\right.$ donnent une sécurité peu supérieure à $1 / 2 \mathrm{~h}$, à $50 \mathrm{l} / \mathrm{s})$,

- le risque que le réservoir ne se remplisse pas (cas des réseaux " éponges ").

Si une ou plusieurs de ces conditions sont réhidibitoires, il peut être intéressant de remplacer le réservoir par un ensemble d'équipements de mesure et de régulation, regroupés dans une chambre de vanne unique (fig. 4).

Ces équipements sont les suivants :

- Vanne de régulation de pression.

Cette vanne assure la coupure hydraulique entre réseau d'adduction et réseau de distribution. Elle assure les fonctions suivantes :

- limitation de la pression aval. Cette fonction peut être différenciée selon que l'on fonctionne le jour ou la nuit,

- ouverture lente pour permettre le remplissage du réseau de distribution après une coupure d'énergie,

- fermeture de sécurité en cas de manque de pression amont.

— Débitmètre électromagnétique.
3. Evolution du coût total actualisé en fonction de la cote piézométrique en tête (réseau de conduites).

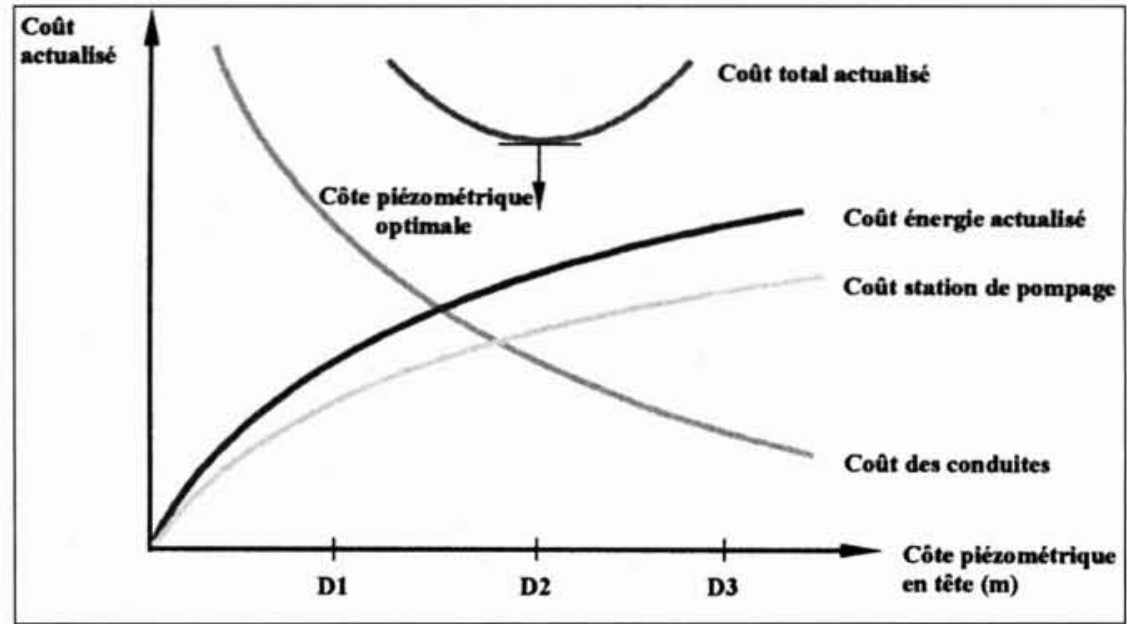




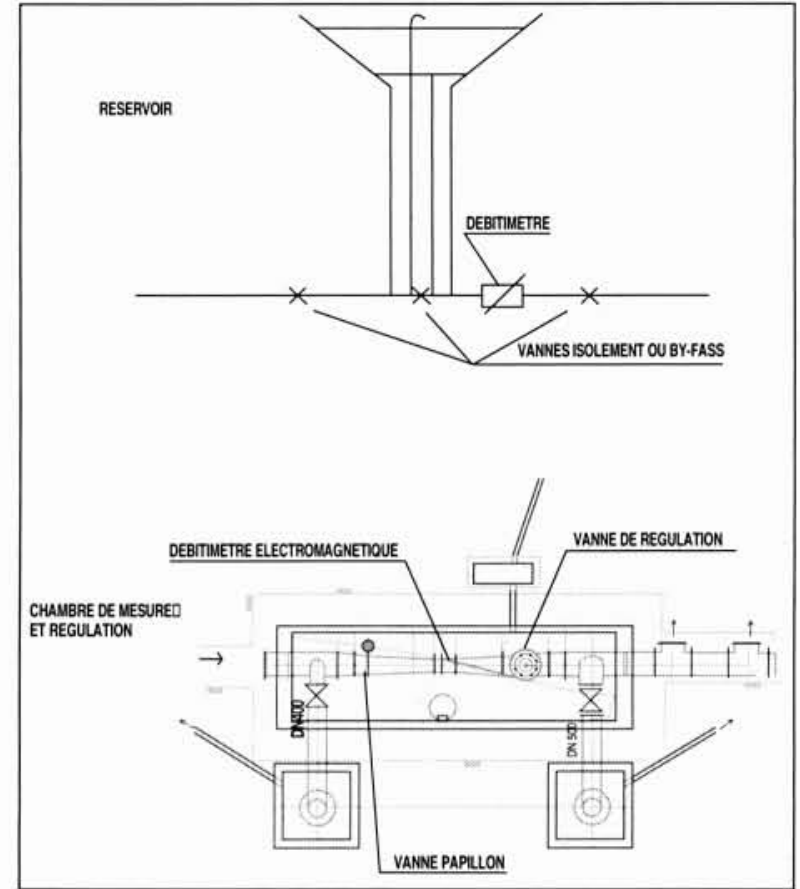

4. Comparaison entre les équipements nécessaires pour un réservoir et une chambre de mesure et de régulation.

Cet appareil assure le comptage des débits de pointe et des volumes distribués; il assure également le contrôle d'un seuil de débit maximum.

- Vanne papillon motorisée.

Elle permet l'isolement des équipements de la chambre de vanne. Grâce à sa motorisation, elle peut se fermer normalement en cas d'arrêt de la station de pompage ou exceptionnellement en cas de détection de survitesse par le débitmètre électromagnétique.

- Des détecteurs de pression amont et aval.

- Des soupapes pour la protection contre les coups de bélier, en cas de mauvais fonctionnement d'une vanne.

A priori, cette solution est plus économique ; il importe cependant de le vérifier, en mettant en balance :

- Le coût d'investissement (réservoir et ses chambres de vannes d'isolement et de mesure amont et aval contre chambre de vanne de mesure et de régulation).
- Dans le cas de l'utilisation de chambre de mesures et de régulation, le supplément d'équipement pour que les pompes puissent distribuer le débit de pointe.

- Dans le cas du réservoir, l'économie d'énergie, s'il permet d'éviter un pompage en heure de pointe d'hiver (si elles existent).

Lorsqu'il n'y a pas de réservoirs de régulation et/ou compensation au niveau de l'entrée des réseaux de distribution, le débit appelé par chacun d'entre-eux se retrouve appelé au niveau de la station de pompage. Au niveau de celle-ci, il faut donc mettre en place un équipement de régulation pour que le débit délivré par les pompes soit équivalent à celui appelé.

\subsection{Régulation au niveau de la station de pompage}

Deux méthodes sont les plus employées : la régulation débitmétrique, la vitesse variable.

La première est la plus économique, puisqu'elle nécessite seulement un débitmètre à la station de pompage (qui doit de toute façon être installé, pour le suivi du fonctionnement).

Par contre, cette solution amène des excès de pression sur les conduites (d'où la nécessité de la fonction " limitation de pression aval » donnée par la vanne de régulation) (fig. 5).

La vitesse variable est plus coûteuse en investissement, mais présente les avantages suivants :

— pas excès de pression,

— possibilité de «suivre " la courbe réseau par une loi du type :

$$
\text { HMT fournie }=\mathrm{A}+\mathrm{B}^{*} \mathrm{Q}^{* * 2}
$$

\subsection{Fonctionnement d'une installation avec chambre de vanne}

En fonctionnement normal, l'appel de débit des utilisateurs se répercute immédiatement à la station de pompage. Au niveau de celle-ci sont mis en marche le nombre de groupes nécessaires, à la vitesse requise s'il $\mathrm{y}$ a des pompes à vitesse variable. Lorsque la pression au niveau d'une chambre de vanne excède la pression acceptable, la vanne de régulation se ferme de façon à maintenir cette dernière sur le réseau de distribution. En fonctionnement de nuit, un réglage de pression plus bas permet de limiter les fuites sur les réseaux.

En cas d'arrêt de la station de pompage, suite à une coupure d'énergie, la vanne papillon se ferme de façon à main-

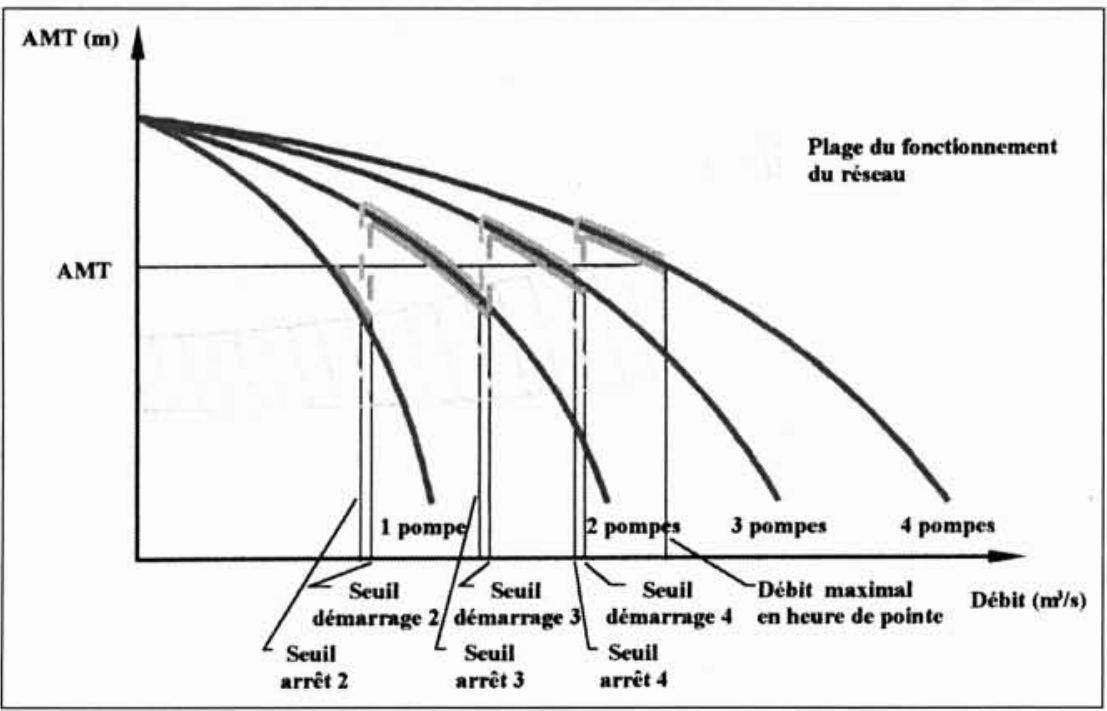

5. Fonctionnement d'une station de pompage ou régulation débitmétrique. 
tenir, au moins, le réseau d'adduction en eau. En cas de panne d'énergie également au niveau d'une ou plusieurs chambres de vanne, c'est la vanne de régulation de pression qui se fermera en lieu et place de la vanne papillon.

Lors du retour du courant, le réseau de distribution se remplira lentement par l'ouverture contrôlée de la vanne de régulation.

\section{VIV LE PROJET DE BUENOS AIRES}

La concession de Buenos Aires au groupement de sociétés entraîné par la Lyonnaise des Eaux est la plus importante jamais accordée dans le monde. Elle consiste à améliorer et développer la distribution pour plus de 10 millions de clients.

Le projet, lié à la mise en œuvre de plus de 250 millions de \$ par an, concerne l'amélioration de la qualité de l'eau traitée, le développement des réseaux d'égouts et l'épuration, la rénovation des réseaux A.E.P. existants, le développement de nouveaux réseaux, en particulier en remplacement de forages existants dans la nappe («plan nitrate $)$.

Parmi tous ces projets, un des plus importants est celui du «Système Ouest ». Il consiste en une ossature hydraulique comprenant (fig. 6):

- la connexion aux conduites existantes issues de la station de traitement géante de San Martin,

- un aqueduc souterrain de $3,5 \mathrm{~m}$ de diamètre et de $15 \mathrm{~km}$ de long réalisé en tunnel à plus de $30 \mathrm{~m}$ de profondeur (d'où son nom de Rio Subterraneo). Le débit maximum transité peut-être de $9 \mathrm{~m}^{3} / \mathrm{s}$,

- deux stations de pompages dans des puits également à 30 mètres de profondeur, dont les débits unitaires sont respectivement de 3 et $5 \mathrm{~m}^{3} / \mathrm{s}$. Ces stations sont appelées 3 de Febrero et Moron (voir figure 6 et 7),

- des réseaux d'adduction au départ de chacune de ces stations. Pour 3 de Febrero, le réseau est en grande partie linéaire et possède 7 départs marqués par des chambres de vanne de mesure et de régulation définies ci-dessus. Pour

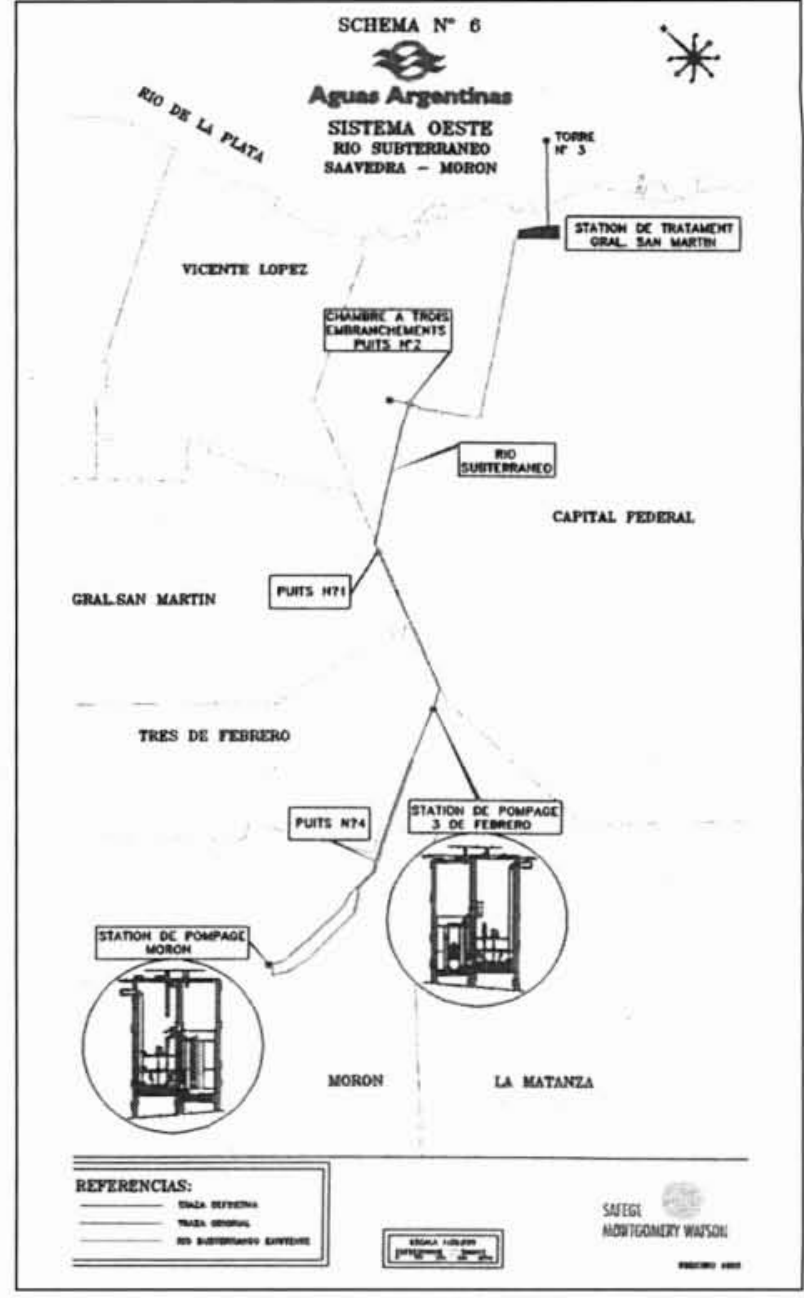

6. Le système ouest.

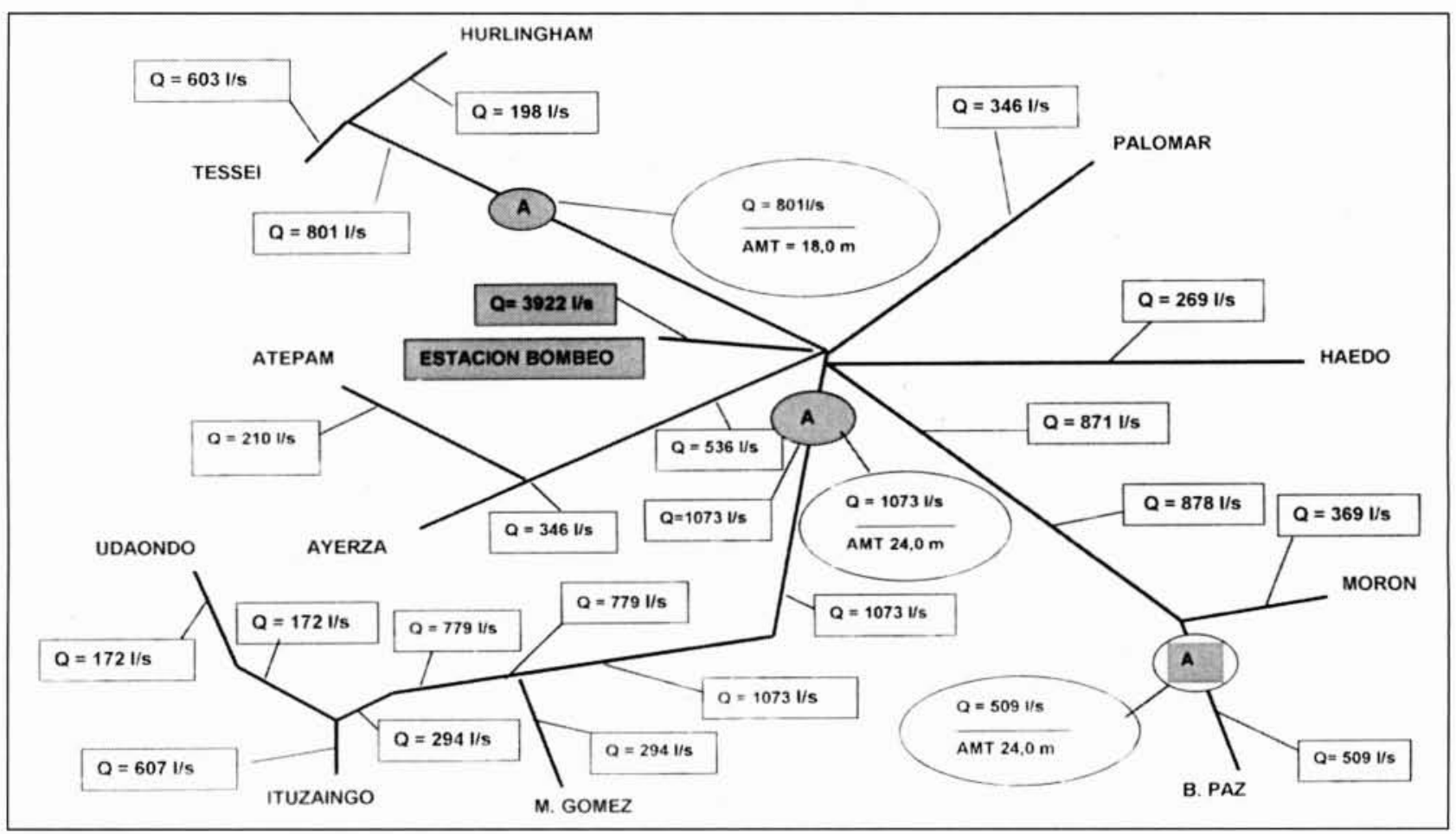

7. Plan du réseau. 
Moron, le réseau est en étoile autour de la station ; il possède 11 départs vers des réseaux de distribution (fig. 7). Les deux projets représentent $50 \mathrm{~km}$ de conduites de 500 à $1300 \mathrm{~mm}$,

- les chambres de vannes de mesure et de régulation (respectivement 7 et 11 ),

- les centaines de kilomètres des réseaux de distribution (la plupart sont existants).

\section{$V \square A P P L I C A T I O N$ DES TECHNIQUES D'OPTIMISATION ET D'ACTUALISA- TION AU PROJET}

Lors de l'établissement des projets de faisabilité des conduites d'adduction, on avait utilisé les méthodes classiques de calcul des diamètres sur la base de critères de vitesse «normales ». Il avait également été prévu une régulation par réservoir surélevé.

Lors de la phase d'étude sommaire, la direction de la Lyonnaise a demandé au bureau d'étude Safege-Montgomery Watson d'examiner toutes les possibilités d'économie sur le projet, sans que la quantité et la qualité de la desserte ne soient affectées. En particulier, il nous a été demandé d'examiner toutes les solutions qui pouvaient permettre de supprimer les réservoirs.

Ce dernier point a été résolu le premier grâce au choix des chambres de mesure et de régulation décrites ci-dessus. Il a été complété par un système de télécontrôle qui assure la transmission à la station de pompage des états et des mesures relevées à chaque chambre de vanne.

L'optimisation du diamètre des conduites d'adduction a été conduite de la manière suivante : détermination de la cote piézométrique optimale au départ de chaque station. Pour les deux réseaux, on a obtenu des cotes de l'ordre de 100 mètres. On notera qu'au niveau faisabilité celles-ci n'étaient que de 70 mètres. L'étude d'optimisation a donc conduit à une réduction notable des diamètres sur certains tronçons.

Mais on a constaté également que, du fait de la structure du réseau d'adduction, certains départs disposaient d'une cote piézométrique excédentaire.

L'optimisation a donc été reprise en introduisant des accélérateurs sur tous ces derniers.

Un accélérateur est un équipement en ligne, pour lequel la cote piézométrique aval est inférieure à la cote piézométrique amont. Il y a donc un débit gravitaire qui est augmenté (accéléré), lorsque la pompe se met en marche. Cet accélérateur ne doit pas être confondu avec un surpresseur pour lequel la cote piézométrique aval est supérieure à la cote amont ; il n'y a donc pas de possibilité de débit gravitaire (fig. 8).

En introduisant les accélérateurs, on a alors constaté que l'on retrouvait la cote initiale de la faisabilité, mais avec des diamètres, cette fois, nettement réduits.

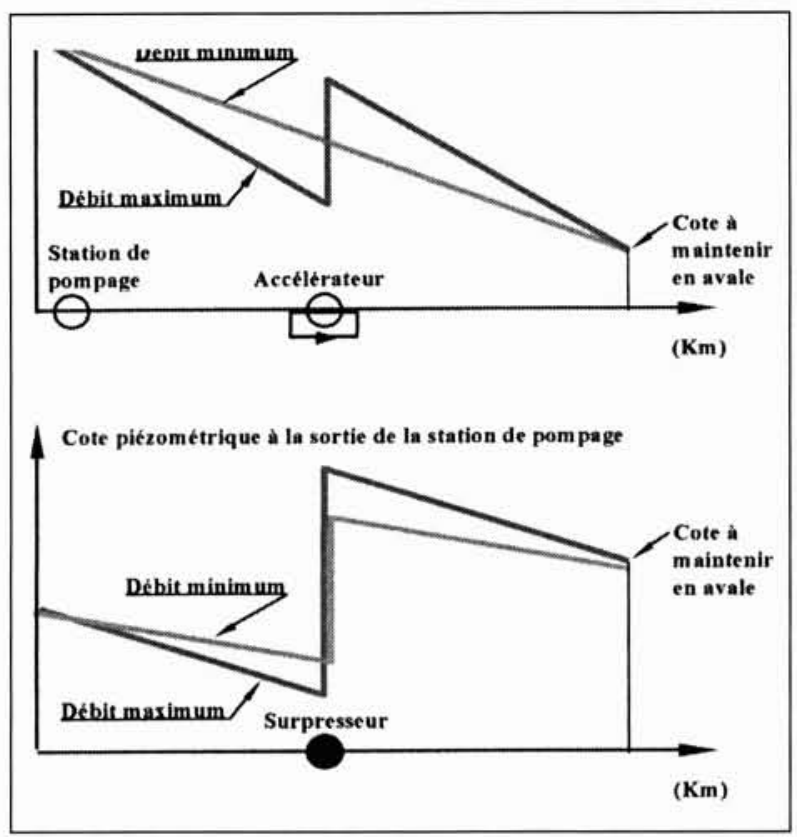

8. Lignes piézométriques avec accélérateur de surpresseur.

De plus, comme ces accélérateurs n'interviennent qu'à partir d'un certain seuil de débit, on s'est aperçu que l'installation de certains d'entre-eux pouvait être différée de plus de 10 ans.

Au final sur un montant d'investissement, prévu lors de la faisabilité, pour les réservoirs + réseau d'adduction, d'environ 58 millions de $\$$, l'économie due à l'optimisation et à la suppression des réservoirs est de l'ordre de 14 millions de \$.

\section{VI $\square$ CONCLUSION}

Dans les projets d'adduction de taille réduite, il n'est pas d'usage de réaliser des études économiques, même simplifiées.

Lorsque les projets prennent de l'ampleur, les résultats obtenus sur l'A.E.P. de Buenos Aires, montrent l'intérêt qu'il y a eu d'avoir à bouleverser les habitudes en utilisant les notions de coûts actualisés et de diamètre optimum.

Sur ce projet, en effet, on a pu à la fois limiter les coûts d'énergie et l'investissement, ce dernier dans une proportion très sensible.

Le remplacement des réservoirs coûteux par des chambres de vanne de régulation a permis la mise en place d'un système de télégestion en temps réel. Ce dernier est un outil formidable d'aide à la gestion au quotidien, comme à la gestion statistique des ouvrages. 